\title{
Keratin 17 is Not Always a Marker of Proliferation of Keratinocytes in Skin Diseases
}

\author{
Li Cui \\ Zirong Zhu ${ }^{2}$ \\ Yiguo Feng ${ }^{3}$ \\ Yanfei Zhang ${ }^{3}$ \\ 'Department of Dermatology, Shaanxi \\ Provincial People's Hospital, Xian, \\ People's Republic of China; ${ }^{2}$ Department \\ of Dermatology, Xi'an People's Hospital, \\ Xian, People's Republic of China; \\ ${ }^{3}$ Department of Dermatology, The \\ Second Affiliated Hospital of Xi'an \\ Jiaotong University, Xian, People's \\ Republic of China
}

Correspondence: Yanfei Zhang

Department of Dermatology, The Second

Affiliated Hospital of Xi'an Jiaotong

University, Xian, People's Republic of

China

Email zhangyanfei723@I63.com
Purpose: Keratin 17 (K17) is an embryonic keratin and overexpression is seen in psoriasis, which is a hyperproliferation skin disease. Nonetheless, whether it is also highly expressed in other proliferative skin diseases remains unclear. The aim of this study is to explore the expression of K17 in cutaneous lichen planus (CLP), lichen simplex chronicus (LSC), and prurigo nodularis (PN).

Methods: A total of 20 skin samples from CLP lesions, 20 from LSC lesions, 20 from PN lesions, and 10 healthy adult skin tissues were obtained. Then, the expression of K17 was analyzed using immunohistochemistry on paraffin-embedded tissue sections. Furthermore, quantitative and semi-quantitative immunohistochemical scores of K17 were independently evaluated under a microscope by 2 dermatologists.

Results: Immunohistochemical analysis revealed that in normal skin, K17 was minimally expressed. Nevertheless, it was highly expressed in all epidermal layers in CLP lesions $(P$-value $<0.01)$, and negatively expressed in LSC and PN lesions (all $P$-value $>0.05$ ). The average gray value of K17 in CLP was $151.153 \pm 13.985$ ( $P$-value $<0.001$ ), while the average values of K17 in LSC and PN were $178.720 \pm 12.001$ and $181.316 \pm 8.920$, respectively (all $P$-value $>0.05$ ).

Conclusion: K17 is potentially expressed in certain inflammatory skin diseases, including psoriasis and lichen planus. Besides, it is not always a marker of hyperproliferation of keratinocytes in skin diseases.

Keywords: keratin 17, proliferation of keratinocytes, inflammatory skin diseases

\section{Introduction}

Keratin 17 (K17) is an embryonic keratin produced by keratinocytes, and it plays a crucial role in regulating fetal epidermal development, skin wound repair, and induced of several inflammatory skin diseases. ${ }^{1,2}$ Researchers recognize that it as a key marker of human epithelial proliferation. ${ }^{3} \mathrm{~K} 17$ is highly expressed in psoriasis, which is a chronic, recurrent, inflammatory skin condition, characterized by hyperproliferation, aberrant differentiation and apoptosis of keratinocytes (KC), and infiltration of inflammatory cells in dermis. ${ }^{4,5}$ Cutaneous Lichen planus (CLP) is a chronic, pruritic, T cell-mediated inflammatory skin disease. ${ }^{6}$ Numerous similarities in pathogenesis have been noted between psoriasis and CLP. ${ }^{7}$ K17 expression occurs in lichen planus lesions, cutaneous wound healing, and warts, therefore, the expression of K17 might reflect hyperproliferative changes. ${ }^{3,8}$ Prurigo nodularis (PN) presents with crusted or excoriated light to bright-red nodules on clinical examination. ${ }^{9}$ Notably, lichen simplex chronicus (LSC) is a skin disorder characterized by lichenification of skin. ${ }^{10}$ Both PN and LSC are chronic pruritic skin 
diseases, with epidermal hyperkeratosis, irregular acanthosis and dermal lymphocytic infiltrate when observed in histopathology. ${ }^{9,10}$ Whether K17 is a real marker of proliferative changes in hyperkeratotic skin disease remains unclear. As such, this study explores the expression of K17 in CLP, PN, and LSC.

\section{Materials and Methods Observed Groups of Persons}

Patients diagnosed with CLP, PN and LSC accompanied by typical clinical manifestations and confirmed by histopathology, and adult normal healthy skin (NHS) as control group were recruited as research objects. All selected patients were not administered with any drugs (including glucocorticoids, retinoic acid, and immunosuppressive drugs) within 2 months, and did not use any topical drugs in the previous 2 weeks. Written informed consent was obtained from all participants. Biopsies were conducted under local anesthesia. All experimental protocols were approved by the Institutional Review Board of the Xi'an Jiaotong University, and performed according to guidelines governing ethics' care in China. This study was performed in accordance with the rules laid down in the Declaration of Helsinki and its later amendments.

\section{Immunohistochemical Analysis}

All tissues were embedded in paraffin and specimen sections were incubated with the K17 primary antibody (monoclonal mouse antibody, clone E3, purchased from Fuzhou Maixin Biotechnology Development Co., Ltd. China) overnight at $4^{\circ} \mathrm{C}$. Then, they were incubated with an appropriate biotinylated streptavidin-horseradish peroxidase-conjugated secondary antibody (ZSGB-BIO, Beijing, China), followed by hematoxylin counterstaining, and finally visualized using 3'-diaminobenzidine (ZSGBBIO, Beijing, China). Phosphate buffered saline (PBS) was used as the primary antibody in negative control.

\section{Semi-Quantitative and Quantitative} Analysis of Immunohistochemical Results

Stained sections were visualized on NanoZoomer Digital Pathology (Hamamatsu, Japan). For quantitative analysis, 5 randomly selected images at high-power fields (200× magnification) were digitally captured using a DP 70 CCD camera (Olympus Corp., Tokyo, Japan). The HPIAS-1000 fully automatic color pathology report analysis system (Wuhan Tongji Qianping Image Engineering Co., Ltd, China) was used to evaluate the average gray value. The average gray value was inversely proportional related to protein expression.

Semi-quantitative analysis of immunohistochemical results: The sample was scored based on the percentage of positive cells and staining intensity score. The staining intensity score was 0 for unstained, 1 for light yellow, 2 for yellow-brown, and 3 for brown. Based on the percentage of positive cells, the score was divided into: 0 points for $<5 \%$, 1 point for $5 \%$ to $25 \%$, 2 points for $26 \%$ to $50 \%$, and 3 points for $>50 \%$. The degree of staining and the percentage of positive cells was multiplied in each section is the final score: 0 to 1 was negative, and $\geq 2$ was positive.

\section{Statistical Analysis}

All data were processed using SPSS13.0 statistical analysis software (SPSS Inc., Chicago, IL, USA). Unpaired $t$-test and Chi-square test were used to analyze the differences. The results were presented as $\mathrm{X} \pm \mathrm{S}, \mathrm{P}<0.05$ was considered as statistically significant.

\section{Results}

\section{The Characteristics of the Participants}

As shown in Table 1, for PN and LSC, young adults accounted for $60-70 \%$ of all the 40 patients, and the male to female ratio was 7 to 3. But CLP was more prevalent among the women. Middle-aged people were predominant and all diseases showed a chronic course.

Table I The Characteristics of the Participants

\begin{tabular}{|l|c|c|c|c|c|c|c|c|}
\hline Clinical Data & N & \multicolumn{3}{|c|}{ Age (Year) } & \multicolumn{3}{c|}{ Sex } & \multicolumn{2}{c|}{ Duration of Disease (Years) } \\
\hline & & $17-25$ & $29-49$ & $>50$ & Man & Female & $<2$ & $>2$ \\
CLP & 20 & 2 & 6 & 12 & 10 & 10 & 18 & 2 \\
PN & 20 & 1 & 12 & 7 & 14 & 6 & 6 & 14 \\
LSC & 20 & 1 & 14 & 5 & 14 & 6 & 8 \\
\hline
\end{tabular}

Abbreviations: CLP, cutaneous lichen planus; LSC, lichen simplex chronicus; PN, prurigo nodularis. 


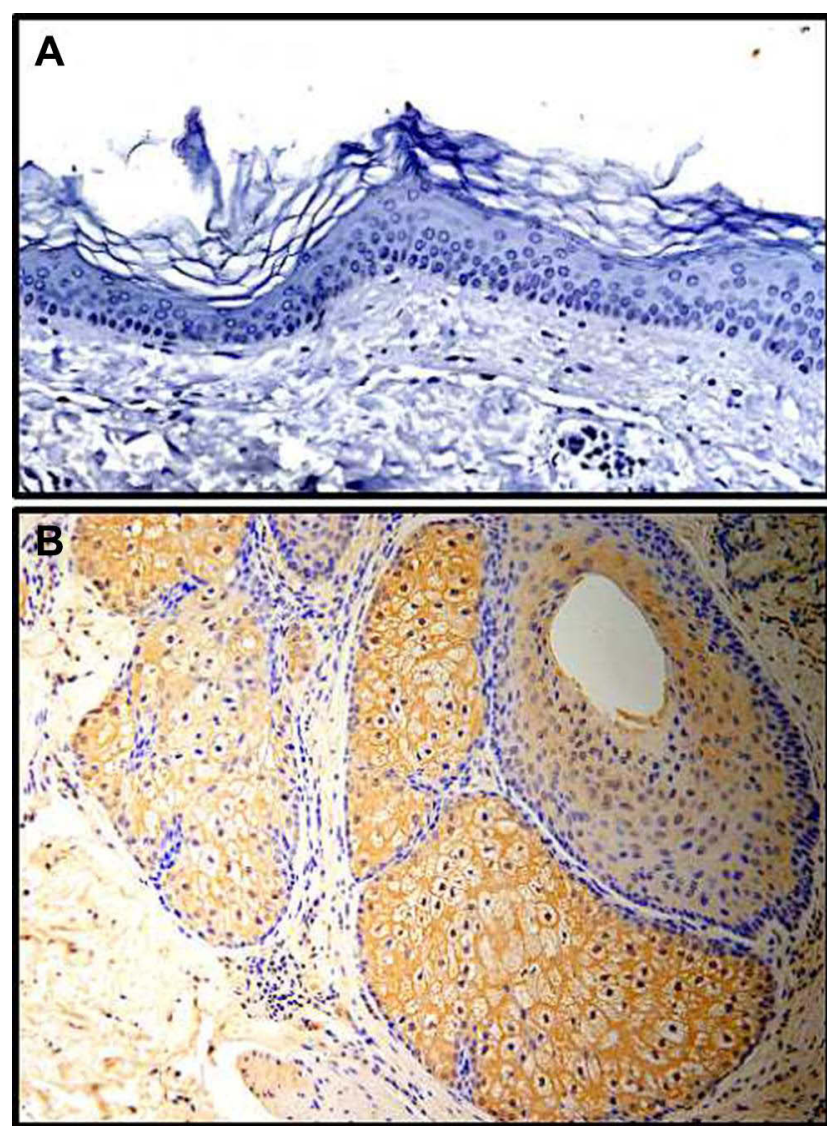

Figure I (A) Immunohistochemistry (IHC) showing KI7 not being expressed in normal healthy skin (NHS); (B) but expressed in hair follicles and sebaceous glands (all magnifications 200x).

\section{KI7 in Normal Healthy Skin}

K17 was minimally expressed in all epidermal layers of normal skin. Only a few basal cells demonstrated weak K17 expression; however, it was primarily distributed in hair follicles and sebaceous glands (Figure 1A and B).

\section{$\mathrm{KI} 7$ in CLP}

Immunohistochemical staining revealed that K17 was highly expressed in all epidermal layers of CLP lesions
(Figure 2A). Among them, 4 cases had weak positive expressed in spinous and granular layers, 12 cases were moderately expressed, while 4 cases were strongly expressed (Table 2). K17 was majorly localized in the cytoplasm of keratinocytes. The average gray value of K17 was $151.153 \pm 13.985(P$-value $<0.001)$ (Table 3 ).

\section{$\mathrm{KI}$ I in PN and LSC}

In lesions of LSC, 1 case was positive stained for K17, with brown particles in the granular and spinous layers. A total of 4 cases were weakly positive expressed for K17, mostly in the basal layer, while 2 cases were suspicious, with few scattered brown particles. The remaining 14 cases were negative (Figure 2B, Table 2). In PN, only 2 skin lesions were weakly positive stained for K17, while others were negative (Figure 2C, Table 2). The average gray values of K17 in LSC and PN were $178.720 \pm 12.001$ and 181.316 \pm 8.920 , respectively (all $P$-value $>0.05$ ). Compared with PN and LSC, the average gray value of K17 in CLP was lower (all $P$-value $<0.001$ ) (Table 3).

\section{Discussion}

K17 belongs to the type I intermediate family of keratins, and it is a vital marker of human epithelial cell proliferation and is implicated in unique organizations of epithelial tissues. ${ }^{3}$ In addition to promoting cell proliferation and growth, K17 can regulate skin inflammation, differentiation and development of skin appendages. Despite not being expressed in normal skin epidermis, K17 is overexpressed in hyperproliferative conditions, including psoriasis, or after sustaining a wound. ${ }^{1,2}$ Here, K17 was minimally expressed in normal epidermis, and mainly presented in the follicles and sebaceous glands, this finding consistent with previous research results. ${ }^{1,2}$

So far, the pathogenesis of LSC and PN are scantily understood. However, reports indicate that dermal and
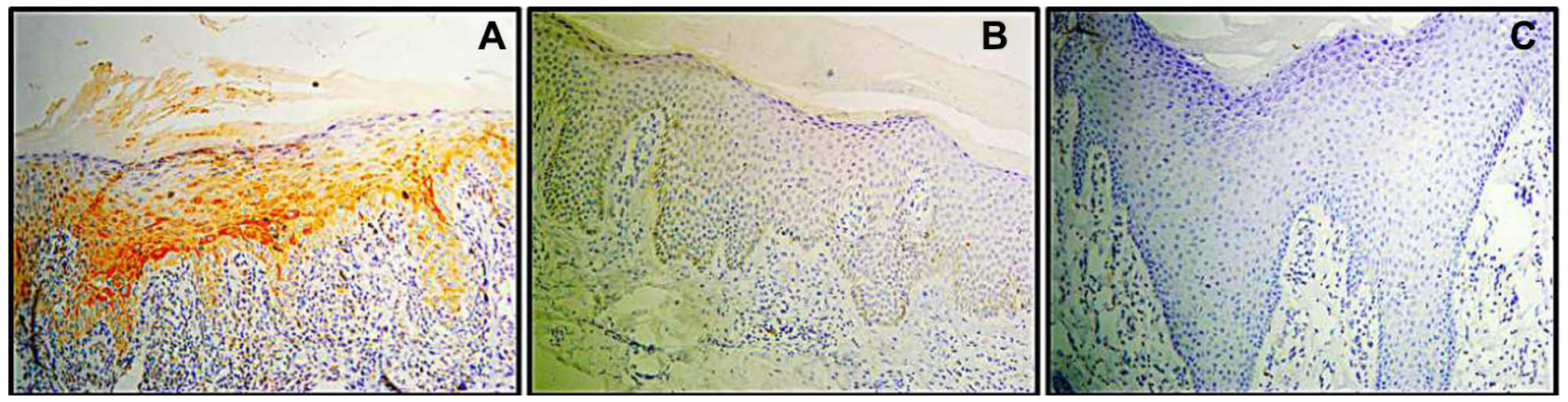

Figure 2 (A) Immunohistochemistry (IHC) demonstrated that KI7 was highly expressed in all epidermal layers in cutaneous lichen planus (CLP) lesions (brown staining); (B) but not in lichen simplex chronicus (LSC); (C) and prurigo nodularis (PN). (all magnifications 200x). 
Table 2 The Expression of KI7 in the Lesions of CLP, LSC and PN

\begin{tabular}{|l|c|c|c|c|c|c|c|}
\hline Samples & N & - & +- & + & ++ & +++ & $P$-value \\
\hline CLP & 20 & 0 & 0 & 4 & 12 & 4 & $<0.01$ \\
LSC & 20 & 14 & 2 & 4 & 0 & 0 & $>0.05$ \\
PN & 20 & 18 & 0 & 2 & 0 & 0 & $>0.05$ \\
NHS & 10 & 9 & 1 & 0 & 0 & 0 & \\
\hline
\end{tabular}

Abbreviations: CLP, cutaneous lichen planus; LSC, lichen simplex chronicus; PN, prurigo nodularis; NHS, normal healthy skin.

Table 3 Quantitative Analysis the Expression of KI7

\begin{tabular}{|l|c|c|c|}
\hline Groups & $\mathbf{N}$ & $\begin{array}{c}\text { Average Gray Value (Mean } \\
\pm \text { Std. Dev.) }\end{array}$ & P-value \\
\hline $\mathrm{NHS}^{1}$ & 10 & $185.565 \pm 4.994$ & ${ }^{\mathrm{a}} P<0.00 \mathrm{I}$ \\
$\mathrm{CLP}^{2}$ & 20 & $151.153 \pm 13.985$ & ${ }^{\mathrm{b}} P<0.00 \mathrm{I},{ }^{\mathrm{c}} P<0.00 \mathrm{I}$ \\
$\mathrm{LSC}^{3}$ & 20 & $178.720 \pm 12.001$ & ${ }^{\mathrm{d}} P=0.4627,{ }^{\mathrm{e}} P=0.1174$ \\
$\mathrm{PN}^{4}$ & 20 & $181.316 \pm 8.920$ & ${ }^{\mathrm{f}} P=0.3284$ \\
\hline
\end{tabular}

Notes: ${ }^{a}$ Comparison between groups I and 2, ${ }^{\mathrm{b}}$ Comparison between groups 2 and 3 , 'Comparison between groups 2 and 4 , 'Comparison between groups 3 and 4 , ${ }^{e}$ Comparison between groups I and $3,{ }^{f}$ Comparison between groups I and 4 .

Abbreviations: CLP, cutaneous lichen planus; LSC, lichen simplex chronicus; PN, prurigo nodularis; NHS, normal healthy skin; Std.Dev., Standard Deviation.

perivascular dense infiltrate with $\mathrm{T}$ cells, mast cells, eosinophil granulocytes, and expression of interleukin-31, which all promote epidermal hyperplasia. ${ }^{11}$ In this work, K17 was not expressed in the hyperplastic epidermis, despite hyperplastic epidermis being a characteristic of LSC and PN. Therefore, the expression of K17 is potentially related to inflammatory factors, it might be in LSC and $\mathrm{PN}$, no inflammatory factors promoting the expression of K17 were found, hence further research is necessary.

In CLP, K17 was highly expressed in all the epidermal layers, its upregulated expression being a precise response to the inflammatory response related to high levels of IFN$\gamma .{ }^{12}$ IFN- $\gamma$ promotes the occurrence and development of lichen planus. ${ }^{13}$ Besides, IFN- $\gamma$ can increase the expression of K17 in human keratinocyte and positive feedback between IFN- $\gamma$ activity and K17 expression in psoriasis was found. ${ }^{12}$ Therefore, it is apparent that K17 was highly expressed in lichen planus and psoriatic epidermis, both being IFN- $\gamma$-induced disorders. Interestingly, K17 has been observed in several types of skin tumors and epidermal malignancies, including keratinizing types of basal cell carcinoma (BCC) or invasive squamous cell carcinoma (SCC), actinic keratosis, Bowen disease, and urothelial neoplasia. ${ }^{14-17}$ Of note, K17 might be a biomarker of cancer diagnosis and prognosis. Besides promoting cell growth, K17 regulates protein synthesis, and being a hallmark of $\mathrm{KC}$ proliferation, it is considered the K17 maybe a therapeutic target. ${ }^{2,18,19} \mathrm{~K} 17$ potentially promotes epithelial proliferation and tumor growth by polarizing the immune response in skin. ${ }^{20}$ As such, K17 is a significantly important factor in inflammatory diseases and tumors.

Our recent studies verified the overexpression of K17 in CLP, a disease attributed to an immune response in the skin. In PN and LSC, epidermal hyperplasia might be induced by itching scratching cycle, not directly caused by inflammatory factors, thereby confirming that K17 was not expressed in PN and LSC.

\section{Conclusion}

Therefore, overexpression of K17 cannot be always regarded as a precise manifestation of hyperkeratosis. It might only be a marker of proliferation of keratinocytes in a few inflammatory skin diseases, including psoriasis and lichen planus, but not always a marker of all proliferation skin diseases.

\section{Funding}

This work was supported by National Natural Science Foundation of China (Number: 81703129).

\section{Disclosure}

The authors report no conflicts of interest in this work.

\section{References}

1. Ide M, Kato T, Ogata K, Mochiki E, Kuwano H, Oyama T. Keratin 17 expression correlates with tumor progression and poor prognosis in gastric adenocarcinoma. Ann Surg Oncol. 2012;19(11):3506-3514. doi:10.1245/s10434-012-2437-9

2. Kim S, Wong P, Coulombe PA. A keratin cytoskeletal protein regulates protein synthesis and epithelial cell growth. Nature. 2006;441 (7091):362-365. doi:10.1038/nature04659

3. Karantza V. Keratins in health and cancer: more than mere epithelial cell markers. Oncogene. 2011;30(2):127-138. doi:10.1038/onc.2010.456

4. Freedberg IM, Tomic-Canic M, Komine M, Blumenberg M. Keratins and the keratinocyte activation cycle. $J$ Invest Dermatol. 2001;116 (5):633-640. doi:10.1046/j.1523-1747.2001.01327.x

5. Jiang M, Sun Z, Dang E, et al. TGFbeta/SMAD/microRNA-486-3p signaling axis mediates keratin 17 expression and keratinocyte hyperproliferation in psoriasis. J Invest Dermatol. 2017;137(10):2177-2186. doi:10.1016/j.jid.2017.06.005

6. Dutz JP. T-cell-mediated injury to keratinocytes: insights from animal models of the lichenoid tissue reaction. J Invest Dermatol. 2009;129 (2):309. doi:10.1038/jid.2008.242

7. Li X, Li J, Yang Y, et al. Differential gene expression in peripheral blood $\mathrm{T}$ cells from patients with psoriasis, lichen planus, and atopic dermatitis. J Am Acad Dermatol. 2013;69(5):e235-e243. doi:10.1016/j. jaad.2013.06.030 
8. Schofield JK, De Berker D, Milligan A, et al. Keratin expression in cutaneous lichen planus. Histopathology. 1995;26(2):153-158. doi:10.1111/j.1365-2559.1995.tb00645.x

9. Pereira MP, Steinke S, Zeidler C, et al. European Academy of Dermatology and Venereology European Prurigo Project: expert consensus on the definition, classification and terminology of chronic prurigo. J Eur Acad Dermatol Venereol. 2018;32(7):1059-1065. doi: $10.1111 /$ jdv. 14570

10. Lotti T, Buggiani G, Prignano F. Prurigo nodularis and lichen simplex chronicus. Dermatol Ther. 2008;21(1):42-46. doi:10.1111/j.1529. 8019.2008.00168.x

11. Ständer S, Yosipovitch G, Legat FJ, et al. Trial of nemolizumab in moderate-to-severe prurigo nodularis. $N$ Engl J Med. 2020;382 (8):706-716. doi:10.1056/NEJMoa1908316

12. Zhang J, Fang H, Wang R, Dang E, Jiang M, Wang G. Effect of calcipotriol on IFN- $\gamma$-induced keratin 17 expression in immortalized human epidermal keratinocyte cells. Med Sci Monit. 2017;23:6049-6056. doi:10.12659/msm.904850

13. Shao S, Tsoi LC, Sarkar MK, et al. IFN- $\gamma$ enhances cell-mediated cytotoxicity against keratinocytes via JAK2/STAT1 in lichen planus. Sci Transl Med. 2019;11(511):eaav7561. doi:10.1126/scitranslmed.aav7561

14. Linskey KR, Gimbel DC, Zukerberg LR, Duncan LM, Sadow PM, Nazarian RM. BerEp4, cytokeratin 14, and cytokeratin 17 immunohistochemical staining aid in differentiation of basaloid squamous cell carcinoma from basal cell carcinoma with squamous metaplasia. Arch Pathol Lab Med. 2013;137(11):1591-1598. doi:10.5858/arpa.2012-0424-OA
15. Lan YJ, Chen $\mathrm{H}$, Chen JQ, Lei QH, Zheng M, Shao ZR Immunolocalization of vimentin, keratin 17, Ki-67, involucrin, $\beta$ catenin and E-cadherin in cutaneous squamous cell carcinoma. Pathol Oncol Res. 2014;20(2):263-266. doi:10.1007/s12253-0139690-5

16. Fernandez-Flores A. Cytokeratin 17 immunoexpression in actinic keratosis (bowenoid and nonbowenoid) and in Bowen disease. Ann Diagn Pathol. 2016;20:1-6. doi:10.1016/j.anndiagpath.2015.11.001

17. Babu S, Mockler DC, Roa-Peña L, et al. Keratin 17 is a sensitive and specific biomarker of urothelial neoplasia. Mod Pathol. 2019;32 (5):717-724. doi:10.1038/s41379-018-0177-5

18. Yang L, Fan X, Cui T, Dang E, Wang G. Nrf2 promotes keratinocyte proliferation in psoriasis through up-regulation of keratin 6 , keratin 16, and keratin 17. J Investig Dermatol. 2017;137(10):2168-2176. doi:10.1016/j.jid.2017.05.015

19. Zhuang Y, Han C, Li B, et al. NB-UVB irradiation downregulates keratin-17 expression in keratinocytes by inhibiting the ERK1/2 and STAT3 signaling pathways. Arch Dermatol Res. 2018;310 (2):147-156. doi:10.1007/s00403-018-1812-1

20. Depianto D, Kerns ML, Dlugosz AA, Coulombe PA. Keratin promotes epithelial proliferation and tumor growth by polarizing the immune response in skin. Nat Genet. 2010;42(10):910-914. doi:10.1038/ng.665

\section{Publish your work in this journal}

Clinical, Cosmetic and Investigational Dermatology is an international, peer-reviewed, open access, online journal that focuses on the latest clinical and experimental research in all aspects of skin disease and cosmetic interventions. This journal is indexed on CAS.
The manuscript management system is completely online and includes a very quick and fair peer-review system, which is all easy to use. Visit http://www.dovepress.com/testimonials.php to read real quotes from published authors. 